\title{
Screening ineffectiveness and THz emission at bare LO phonon frequencies
}

\author{
F. M. Souza and J. C. Egues \\ Departamento de Física e Informática, Instituto de Física de São Carlos, \\ Universidade de São Paulo, 13560-970 São Carlos, São Paulo, Brazil.
}

(Dated: October 30, 2018)

\begin{abstract}
Within a hydrodynamic approach we investigate the dynamics of an inhomogeneous electron-hole gas coupled to phonons in Te and the corresponding $\mathrm{THz}$ emission. We find that the longitudinal inhomogeneity of the photogenerated electron-hole gas - due to short absorption lengths in Te gives rise to screening ineffectiveness for non-zero wave-vector modes. This allows for $\mathrm{THz}$ dynamics and emission at the bare LO phonon frequency $\nu_{L O}$ even at high carrier densities. Screening ineffectiveness thus provides an appealingly simple explanation for the existence of bare modes at $\nu_{L O}$ in longitudinally inhomogeneous systems such as Te; no lateral inhomogeneity of the excitation spot is needed here.
\end{abstract}

Terahertz-range dynamics of non-equilibrium carriers interacting with phonons is a very active field of research in semiconductors. 1 Pump-and-probe timeresolved spectroscopies with ultrashort laser pulses have played a crucial role in unveiling many interesting features in these complex systems. The appearance of time-dependent electric fields following the pump excitation - due to screening of depletion-layer fields, chargeseparation fields, lattice polarization fields, etc. - gives rise to electro-optic induced reflectivity/transmittance changes and terahertz emission due to dipole radiation. Experimentalists probe the system in essentially two ways: by measuring (i) the reflectivity/transmittance changes of a probe pulse and/or (ii) the emitted radiation. These canonical experimental geometries yield, in principle, complementary information on the system characteristic frequencies.

In $n$-doped GaAs, for instance, the usual two branches $L_{+}$and $L_{-}$describing the long wavelength plasmonphonon coupled modes as a function of the carrier density are easily mapped out from electro-optic modulation experiments. 2 As pointed out in Ref. 3, the $L_{+}$mode is usually interpreted as a bare LO phonon mode $\nu_{L O}$ at low carrier densities, while the $L_{-}$mode is referred to as a screened LO phonon mode $\nu_{T O}$ at high carrier densities. Interestingly enough, beating of these TO and LO modes is observed in the experiments. This is due to the simultaneous excitation of these modes due to the lateral inhomogeneity of the pump pulse which generates regions of high and low carrier densities - sampled simultaneously by the probe pulse. In contrast, terahertz emission spectroscopy in GaAs provides information only on the $L_{-}$ branch at low densities; $L_{+}$and LO phonon frequencies $(>8 \mathrm{THz})$ are not accessible with the current sfectral range $(\lesssim 7 \mathrm{THz})$ of the state-of-the-art antennas.

In Tellurium on the other hand, both reflectivity and $\mathrm{THz}$ emission measurements can probe coupled-mode frequencies. These experiments are performed in both the $c_{\|}$and $c_{\perp}$ geometry. These denote the either parallel or perpendicular orientation of the $\mathrm{c}$ axis of the hexagonal lattice of Te with respect to the excitation surface. Oscillations at bare $\nu_{L O}$ are seen in both experiments for the $\mathrm{c}_{\|}$geometries $\mathrm{G}$ In the $\mathrm{c}_{\perp}$ geometry only $\mathrm{THz}$ emission is possible; a peak at $\nu_{L O}$ is also evident 6 , 7 Similarly to GaAs, the existence of these peaks in the Te response can in principle be explained via the lateral inhomogeneity of the excitation spot. There is, however, a more appealing explanation we propose in this work.

In this letter we theoretically investigate the coupled dynamics of electron-hole-phonon modes in Te and the corresponding $\mathrm{THz}$ emission. Our semiclassical description incorporates phonons and its coupling to carriers via polarization charges in Poisson's equation. Our main finding is the observation that the intrinsic longitudinal inhomogeneity of the photogenerated electron-hole plasma - due to the short absorption lengths in $\mathrm{Te}-$ leads to screening ineffectiveness for non-zero wave vectors. Screening ineffectiveness allows for dynamics and terahertz emission at bare LO phonon frequencies - even at high carrier densities - thus providing a possible alternate/complementary explanation for the observed bare $\mathrm{LO}$ modes in the $\mathrm{THz}$ response of Te. We suggest a simple experiment to tell apart lateral- $(q=0)$ and longitudinal-inhomogeneity $(q \neq 0)$ contributions. A dielectric function analysis (Debye Hückel and RPA) corroborates our numerical findings.

Ingredients. Any reasonable attempt to describe pump-and-probe $\mathrm{THz}$ emission experiments has to necessarily address the coupled-field dynamics of electrons, holes, and phonons. Our semiclassical approach combines (i) moments of the Boltzmann equation describing charge and current densities, (ii) a phenomenological driven-harmonic-oscillator equation simulating LO phonons, and (iii) Poisson's equation providing electronand hole-phonon coupling via polarization charges.

Model. Our one-dimensional model allows motion only perpendicular to the relevant semiconductor surface being excited. We consider carriers with isotropic masses $m_{e}^{*}=0.06 m_{0}$ and $\eta_{h}^{*}=0.114 m_{0}$ (and charges $q_{e}$ and $q_{h}$ ) in parabolic bands. ${ }^{*}$ We assume the electrons and holes are thermalized to distinct constant temperatures $T_{e}$ and $T_{h}$, respectively 10 these are, however, different from the lattice temperature. By considering the semiclassical Boltzmann equation for the distribution function within the usual relaxation-time approximation, we derive equations for both particle and particle current 
densities of electrons $(e)$ and holes $(h), n_{e(h)}(x, t)$ and $J_{e(h)}(x, t)=n_{e(h)}(x, t) v_{e(h)}(x, t)$, respectively; $v_{e(h)}(x, t)$ denotes the electron (hole) mean velocity. These are

$$
\frac{\partial n_{e(h)}(x, t)}{\partial t}+\frac{\partial J_{e(h)}(x, t)}{\partial x}=G(x, t),
$$

and

$$
\begin{aligned}
\frac{\partial J_{e(h)}(x, t)}{\partial t}= & -\frac{\partial}{\partial x} \frac{J_{e(h)}^{2}(x, t)}{n_{e(h)}(x, t)}+\frac{q_{e(h)}}{m_{e(h)}^{*}} n_{e(h)}(x, t) E(x, t)- \\
& \frac{k_{B} T_{e(h)}}{m_{e(h)}^{*}} \frac{\partial}{\partial x} n_{e(h)}(x, t)-\frac{J_{e(h)}(x, t)}{\tau_{e(h)}}
\end{aligned}
$$

where $G(x, t)$ is the generation term (laser). The electric field $E(x, t)$ in Eq. (2) is calculated via Poisson's equation

$$
\frac{\partial E}{\partial x}=\frac{1}{\varepsilon_{0} \epsilon_{\infty}}\left(q_{h} n_{h}+q_{e} n_{e}\right)-\frac{\omega_{T O} \sqrt{\varepsilon_{0}\left(\epsilon_{0}-\epsilon_{\infty}\right)}}{\varepsilon_{0} \epsilon_{\infty}} \frac{\partial w}{\partial x},
$$

which accounts for both electron and hole charge densities and lattice polarization charges $\sim \partial w(x, t) / \partial x$. We assume the vibrational coordinate $w(x, t)$ in Poisson's equation obeys

$$
\frac{\partial^{2} w}{\partial t^{2}}+\frac{1}{\tau_{p h}} \frac{\partial w}{\partial t}+\omega_{T O}^{2} w=\omega_{T O} \sqrt{\varepsilon_{0}\left(\epsilon_{0}-\epsilon_{\infty}\right)} E,
$$

which is driven by the total electric field due to electronhole charge separation and phonon polarization charges. Note that Eq. (州) describes longitudinal oscillations. We account for damping of electrons, holes and phonons via proper relaxation times $\left[\tau_{e}, \tau_{h}\right.$ and $\tau_{p h}$, respectively].

Equation (11) is the continuity equation for electrons or holes; laser pumping of carriers is accounted for by the generation term (gaussian in time and exponentially attenuated in $x$ ). Equation (2) states momentum conservation [the momentum density is $p_{e(h)}(x, t)=$ $\left.n_{e(h)}(x, t) m_{e(h)}^{*} v_{e(h)}(x, t)\right]$. Implicit in (2) is the assumption of thermalized carriers with average energies $\left\langle u_{x}\right\rangle_{e(h)}=\frac{1}{2} k_{B} T_{e(h)}+\frac{1}{2} m_{e(h)}^{*} v_{e(h)}^{2}(x, t)$. With this assumption we are essentially truncating the sequence of equations for higher-order moments. By coupling Eqs. (3) and (4), we extend the phenomenological description of Born and Huang 11 in that we allow the internal electric field to drive lattice vibrations and vice versa. Our description here generalizes the previous investigations of Kuznetsov and Stanton 9 who treat homogeneous systems and introduce averaging over the spot profile and Dekorsy et al 6 who use a drift-diffusion type model.

Numerical solution. The set of six coupled differential equations discussed above is tricky to solve numerically. By carefully using standard explicit schemes for the propagation of partial differential equations, 12 we are able to obtain $n_{e}(x, t), J_{e}(x, t), n_{h}(x, t), J_{h}(x, t), E(x, t)$, and $w(x, t)$ in a wide range of system parameters. As we discuss next, both the radiated signal and the reflectivity response are related to $E(x, t)$.
Emitted THz radiation. The longitudinal charge oscillations in the system are essentially electric dipoles and thus emit electromagnetic waves. Within the dipole approximation, the emitted signal is proportional to the second time derivative of the internal electric field. We define the radiated field $E_{r a d}$ by

$$
E_{r a d} \propto \frac{\partial^{2}}{\partial t^{2}} \int_{0}^{\alpha^{-1}} e^{-\alpha x} E(x, t) d x
$$

which accounts for oscillating dipoles, weighed by the absorption profile, from within only the relevant absorption length $\alpha^{-1}$. Note that the radiated field $E_{\text {rad }}$ is $p$ polarized.

Figures 1(a) and 1(b) show the calculated emitted signals and their Fourier transform, respectively, for several excitation densities $n_{e x c}$, at fixed electron and hole temperatures, $T_{e}=2000 \mathrm{~K}$ and $T_{h}=1050 \mathrm{~K}$ [see also inset in Fig. 1(c)]. The time-dependent signals 1(a) show an initial rise and a dip followed by small oscillations [similar to the experimental detected signal in the inset]. As shown in 1(b), these small oscillations have frequencies close to that of the LO phonon in Te, $\nu_{L O} \sim 2.82 \mathrm{THz}$ ( $c_{\perp}$ geometry); the broad-band peak in $1(\mathrm{~b})$ is due to electron-hole charge separation as discussed in 6 .

The detected theoretical signals in 1(c) are convolutions of the emitted signals in 1(b) and the antenna response [dotted line in 1(b)]. The convoluted signals 1(c) are very similar to the recent data in 1 (d) from Ref. 6. In particular both theoretical and experimental spectra display a broad-band peak followed by a dip at the screened LO frequency $\nu_{T O} \sim 2.6 \mathrm{THz}$ and a subsequent peak at the bare LO phonon frequency $\nu_{L O} \sim 2.82 \mathrm{THz}$. Note that the signals get stronger with increasing excitation densities [curves (1) - (6)]. This intensity enhancement of the signals follows from the amplitude increase of the internal fields with $n_{\text {exc }}$. While the spectral features at the phonon frequencies hardly move with increasing excitation densities, the broad-band features slightly shift to higher frequencies. This shift is due to the faster electronhole charge dynamics at higher densities. 6 The relative intensities of the bare LO peak and that of the broadband peak strongly depend on the antenna response.

Antenna response. We assumed a simplistic antenna response with a high sensitivity around $0.5 \mathrm{THz}$ dropping essentially to zero above $3 \mathrm{THz}$ [dotted line in 1(b)]. This response weighs up the contribution of the broad-band peak in contrast to that of the bare LO phonon. The detected/convoluted signals 1(c) are then "distorted" thus displaying weaker LO phonon peaks [cf. 1(b) and 1(c)].

Normal mode analysis. By linearizing our equations and neglecting damping for simplicity, we obtain the system dielectric function

$$
\epsilon(q, \omega)=\epsilon_{\infty}+\frac{\epsilon_{0}-\epsilon_{\infty}}{1-\left(\frac{\omega}{\omega_{T O}}\right)^{2}}-\sum_{i=e, h} \frac{\omega_{p l, i}^{2} \epsilon_{\infty}}{\omega^{2}-c_{i}^{2} q^{2}},
$$


where $\omega_{p l, i}=\sqrt{4 \pi e^{2} n / \epsilon_{\infty} m_{i}^{*}}, c_{i}=\sqrt{k_{B} T_{i} / m_{i}^{*}}$, and $n$ is the carrier densities. Equation (6) contains both phonon and electron-hole plasma contributions; these are the second and third terms, respectively. The LO phonon contribution is dispersionless. The plasmon contribution contains non-zero wave vector modes which account for the longitudinal inhomogeneity of the electron-hole gas; this term is equivalent to the Debye-Hückel screening (classical). 13 The zeros of the dielectric function define the normal modes of the system: $\epsilon(q, \omega)=0 \Rightarrow \omega(q)$.

Figure 2(a) displays the coupled carrier-phonon modes (solid lines) of the system in the long wavelength limit $(q=0)$ as a function of the carrier density. The branches $L_{+}$and $L_{-}$arise from the anticrossing of the $L \rho$ and plasmon modes (dashed line) due to coupling. 14 Note that for increasing $n$ the $L_{-}$branch tends to the screened LO phonon frequency $\nu_{T O}$. Note that screening is the strongest for $q=0$ and high densities [Eq. (6)]. In the low-density limit, in contrast, $L_{+}$converges to the bare LO frequency $\nu_{L O}$.

Figure 2(b) shows the wave-vector dependence of three particular $L_{-}$frequencies corresponding to $n=2,4$, and $10 \times 10^{17} \mathrm{~cm}^{-3}$. Here as $q$ increases the frequency of these three modes tend to the bare $\nu_{L O}$ value. This interesting feature is dye to screening ineffectiveness for non-zero wave vectors, 15 i.e., for large $q$ values screening by the electron-hole gas becomes ineffective. Equation (6) clearly shows the electron-hole contribution to $\epsilon(q, \omega)$ gets suppressed for increasing $q$. In addition, we find that a more rigorous description of screening within the Random Phase Approximation corroborates our semiclassical normal-mode analysis [see dashed lines in Fig. 2(b)]. We consider only the static limit for simplicity 16 In this limit and for the high temperatures used, the RPA calculation is straightforward.17

The mode analysis above makes it clear that the physics behind the bare LO phonon mode in our numerical results (particularly at high densities, Fig. 1) is that of screening ineffectiveness. We believe this peak at $\nu_{L O}$ is an $L_{-}$unscreened $q \neq 0$ mode; not a low-density $L_{+}$ mode with $q=0$. Note that this $q \neq 0$ phonon-like mode is not affected by the strong damping of the electron-hole gas $\left(\tau_{e(h)}=10\right.$ fs for Te; $\left.{ }^{6}\right)$ its damping constant is actually that of the long-lived phonon modes (we assume $\tau_{p h}=4 \mathrm{ps}$. I) Interestingly enough, the "dip" at $\nu_{T O}$ in Fig. 1 follows from $\epsilon(q, \omega) \rightarrow \infty$ as $\omega \rightarrow \omega_{T O}$ which leads to a suppression of the internal electric field. This suppression is not complete in our simulation because of damping.

Electro-optic modulation signal. The relevant "probe signal" in a pump-and-probe electro-optic modulation experiment is the quantity $S(t) \propto \frac{\partial}{\partial t} \int_{0}^{\alpha^{-1}} e^{-\alpha x}[\gamma E(x, t)$ $\beta w(x, t)] d x$, where $\beta$ and $\gamma$ are (electro-optic) constants.
Figure 3 shows the Fourier spectrum of $S(t)$ for an $n$ doped GaAs system 18 with an arbitrarily-chosen small absorption length $\alpha^{-1}=10 \mathrm{~nm}$ and no depletion layer electric field.19 In this case longitudinal inhomogeneity is relevant. Similarly to the $\mathrm{THz}$ emission signal, here we also clearly see a peak near the bare LO phonon mode. This feature is again due to screening ineffectiveness 20 [see Fig. 2(b)]. Note that the reflectivity spectrum is qualitatively different from the emission one. No dip at the screened LO frequency $\nu_{T O}$ should be expected in the reflectivity spectrum when both the phonon and internal electric fields contribute to the response $(|\beta / \gamma|>1$ in GaAs). In this case, the response does not vanish when $E(x, t)$ is suppressed. In our model, no lateral averaging over the pump spot is needed in order to obtain peaks at $\nu_{T O}$ and $\nu_{L O}$.

Lateral vs longitudinal inhomogeneity. A pump-andprobe reflectivity measurement with an excitation spot much larger than the probe one 21 (for Te a $\mathrm{c}_{\|}$geometry should be used) can conclusively discern contributions from these distinctive inhomogeneities. By focusing the probe right on the high-density central region of the excitation spot, one should see both bare and screened LO phonon peaks in the probe spectral response. While the screened LO peak would represent the $L_{-}$mode with $q=0$ [Fig. 2(a)], the bare LO peak should follow from screening ineffectiveness due to $q \neq 0$ modes [Fig. 2(b)]. The presence of this bare phonon mode at high densities would constitute a unique signature for the relevance of the longitudinal-inhomogeneity effect we propose in this paper. Multiple quantum wells and super|attices are also suitable systems to search for this effect;22 the periodic modulations along the growth direction give rise to longitudinal inhomogeneity. Recent reflectivity experiments in GaAs quantum-confined geometries 3 have found both screened and bare LO modes; these experiments, however, use comparable pump and probe spot sizes.

We have studied the coupled dynamics of electrons, holes and phonons within a hydrodynamic approach. In longitudinally inhomogeneous systems such as Te, we find that screening ineffectiveness at non-zero wave vectors allows for oscillations at the bare LO phonon frequency in both reflectivity and terahertz emission spectra. We believe this mechanism provides an appealingly simple (alternate/complementary) explanation for the existence of these bare LO modes even at high densities.

This work was supported by Fundação de Amparo à Pesquisa do Estado de São Paulo (FAPESP). The authors acknowledge discussions with L. Ioriatti, A. V. Kuznetsov, T. Dekorsy and G. Cho. F.M.S. gratefully acknowledges a fellowship from FAPESP/CAPES. The authors thank M. P. Hasselbeck for pointing out Ref. [4] to them. 
${ }^{2}$ G. C. Cho et al., Phys. Rev. Lett. 77, 4062 (1996); M. Hase et al., J. Lumines. 76\&77, 68 (1998); R. Kersting et al., Phys. Rev. B 58, 4553 (1998); W. Fischler et al., Semicond. Sci. Technol. 15, 813 (2000); See Ref. 11 for a more thorough list.

${ }^{3}$ K. J. Yee, et al. Phys. Rev. B 60, R8513 (1999).

4 For $\mathrm{THz}$ emission measurements using a Si bolometer, see J. N. Heynman et al., Phys. Rev. B 64, 085202 (2001)

${ }^{5}$ T. Dekorsy et al. Phys. Rev. Lett. 74, 738 (1995).

6 T. Dekorsy et al. Phys. Rev. B 53, 4005 (1996).

7 M. Tani et al. J. Appl. Phys. 83, 3473 (1998).

8 The diameter of the excitation spot is much greater than the absorption length.

9 A. V. Kuznetsov and C. J. Stanton [Phys. Rev. B 51, 7555 (1995)] discuss intervalley transfer ("non parabolicity") in a related model.

10 For typical carrier excess energiens, we choose the temperatures so that $T_{e} / T_{h}=m_{h} / m_{e}$.

11 See Max Born and Huang Kun, Theory of Crystal Lattices, The International Series of Monographs on Physics, (Oxford University Press, Oxford, 1985), $2^{\text {nd }}$ edition, Ch. II, p. 104.

12 See, for instance, W. H. Press, S. A. Teukolsky, W. T. Vetterling e B. P. Flannery, Numerical Recipes in $C$, the Art of Scientific Computing, (Cambridge University, London, 1996) $2^{\text {nd }}$ edition, Ch. 19.

13 G. Rickayzen, Green's Functions and Condensed Matter, (Academic Press, New York, 1991), $2^{\text {nd }}$ edition, Ch. 5, p. $121-125$.

14 A. Mooradian and A. L. McWhorter, Phys. Rev. Lett. 19, 849 (1967).

15 See R. A. Cowley and G. Dolling, Phys. Rev. Lett. 14, 549 (1965) for a similar effect in the context of optical modes in ionic crystals.

16 Static RPA does not describe plasmons; hence the small deviation in Fig. 2(b) as $q \rightarrow 0$ (cf. dashed and solid lines). See M. P. Hasselbeck and P. M. Enders, Phys. Rev. B 57, 9674 (1998) for dynamic RPA calculations including nonparabolicity effects; see also F. M. Souza and J.C. Egues, Braz. J. Phys. 32(2A), 415 (2002).

17 Hisanao Sato and Yoshikazu Hori, Phys. Rev. B 36, 6033 (1987).

18 We do not find plasmon modes in undoped systems (Te).

19 Here we have in mind a clean UHV-cleaved surface (no band bending due to Fermi-level pinning).

${ }^{20}$ Interestingly enough, this finding is consistent with earlier Raman spectroscopy data for UHV-cleaved $n$-GaAs samples [H. J. Stolz and G. Abstreiter, J. Vac. Sci. Technol. 19, 380 (1981)]; however, the mechanism we propose here is completely different.

21 A similar experiment has been performed in bulk GaAs [see
G. C. Cho, Ph.D. dissertation, RWTH, Aachen (1997)]: only a screened LO peak is seen in the central high-density region of the spot. This is not surprising since $\alpha^{-1}$ is large in GaAs $(\sim 1 \mu \mathrm{m}$ at $1.47 \mathrm{eV})$ thus making longitudinal inhomogeneity small.

22 See J. J. Baumberg and D. A. Williams, Phys. Rev. B 53, R16140 (1996) for a single-interface geometry.

FIG. 1: Calculated THz signals for differing excitation densities, in time (a) and frequency domains (b), (c), and (d). The emitted spectra (b) display features at lower frequencies due to electron-hole separation and at the characteristic phonon frequencies $\nu_{T O}$ and $\nu_{L O}$. The theoretical detected spectra (c) is obtained by convoluting the emitted signals and the antenna response [dotted line in (b)]. The calculated (c) and experimental (d) detected spectra are very similar: for increasing excitation densities they both present a strong broad band followed by a dip at the TO phonon frequency and a weaker peak at the bare LO phonon frequency. Note also that the signals get stronger for increasing excitation densities. Observe that the relative intensities of the broad peak and that of the LO peak are reversed after convolution [cf. (b) and (c)]. Surprisingly enough, a bare LO mode survives even at high densities $n \sim 5 \times 10^{18} \mathrm{~cm}^{-3}$; in our model this follows from screening ineffectiveness for non-zero wave vectors arising from the intrinsic electron-hole longitudinal inhomogeneity in Te. This effect does not qualitatively change with $T_{e} / T_{h}$ [see inset in (c)]. The inset in (d) shows the experimental time-dependent detected signal.

FIG. 2: Electron-hole-phonon coupled modes. The $q=0$ modes (a) show the usual two branches $L_{-}$and $L_{+}$due to anticrossing. Here screening is operative thus making $\nu_{L O} \rightarrow$ $\nu_{T O}$ for high densities. Screening ineffectiveness is clearly seen in (b) for non-zero wave vector modes: as $q$ increases the $L_{-}$branch approaches the bare $\nu_{L O}$ frequency. Both DebyeHückel and RPA show this trend, which is consistent with our numerical findings.

FIG. 3: Calculated reflectivity spectra for an $n$-doped system with short absorption length. Illustratively we use GaAs with an arbitrarily small $\alpha^{-1}$. Note that both $\nu_{T O}$ and $\nu_{T O}$ are present in the response. No averaging over the spot profile is performed. 

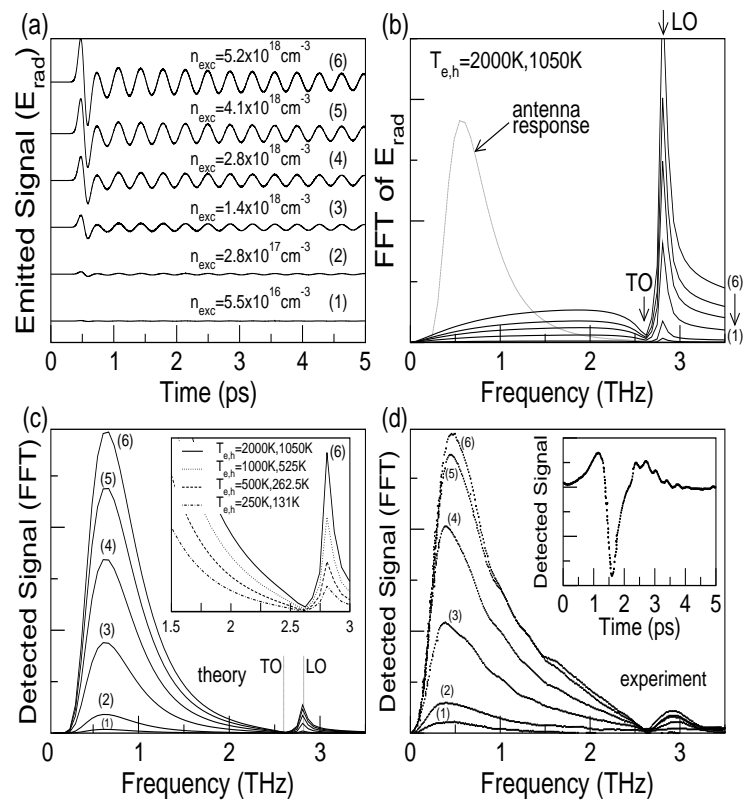

Fig. 1 (Souza and Egues) 


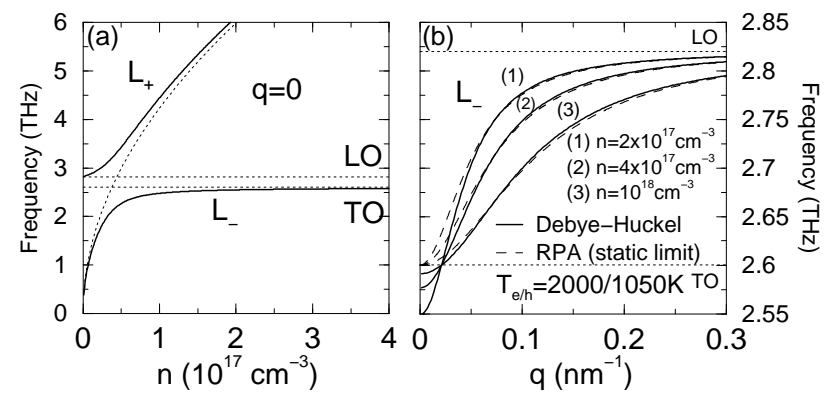

Fig. 2

(Souza and Egues) 


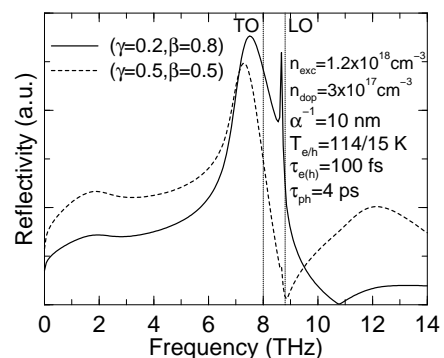

Fig. 3

(Souza and Egues) 